\title{
5-Aminolevulinic acid-mediated sonosensitization of rat RG2 glioma cells in vitro
}

\author{
Krzysztof Bilmin ${ }^{1}$, Tamara Kujawska², Wojciech Secomski ${ }^{2}$, Andrzej Nowicki ${ }^{2}$, Paweł Grieb ${ }^{1}$ \\ ${ }^{1}$ Mossakowski Medical Research Centre, Polish Academy of Sciences, Warsaw, ${ }^{2}$ Institute of Fundamental Technological Research, \\ Polish Academy of Sciences, Warsaw, Poland
}

\begin{abstract}
Sonodynamic therapy (SDT) is a promising technique based on the ability of certain substances, called sonosensitizers, to sensitize cancer cells to non-thermal effects of low-energy ultrasound waves, allowing their destruction. Sonosensitization is thought to induce cell death by direct physical effects such as cavitation and acoustical streaming as well as by complementary chemical reactions generating oxygen free radicals. One of the promising sonosensitizers is 5-aminolevulinic acid (ALA) which upon selective uptake by cancer cells is metabolized and accumulated as protoporphyrin IX. The objective of the study was to describe ALA-mediated sonodynamic effects in vitro on a rat RG2 glioma cell line. Glioma cells, seeded at the bottom of 96-well plates and incubated with ALA $(10 \mu \mathrm{g} / \mathrm{ml})$ for $6 \mathrm{~h}$, were exposed to the sinusoidal US pulses with a resonance frequency of $1 \mathrm{MHz}, 1000 \mu \mathrm{s}$ duration, 0.4 duty-cycle, and average acoustic power varying from $2 \mathrm{~W}$ to $6 \mathrm{~W}$. Ultrasound waves were generated by a flat circular piezoelectric transducer with a diameter of $25 \mathrm{~mm}$. Cell viability was determined by MTT assay. Structural cellular changes were visualized with a fluorescence microscope. Signs of cytotoxicity such as a decrease in cell viability, chromatin condensation and apoptosis were found. ALA-mediated SDT evokes cytotoxic effects of low intensity US on rat RG2 glioma cells in vitro. This cell line is indicated for further preclinical assessment of SDT in in vivo conditions.
\end{abstract}

Key words: 5-aminolevulinic acid, sonodynamic therapy, rat RG2 glioma cells, cell viability.

\section{Introduction}

Sonodynamic therapy (SDT) is a new treatment modality of solid cancers in the early preclinical development phase. The idea of SDT stems from photodynamic therapy (PDT) in which photo-sensitizer substances excited by light produce the avalanche of cytotoxic reactive oxygen species that kill cancer cells. Unlike PDT, SDT uses low-intensity ultrasound (US) waves to kill cells. A low-intensity ultrasound may be defined as US which does not produce hyperthermia which would be directly cytotoxic $\left(>43^{\circ} \mathrm{C}\right)$. To obtain cell killing by low-intensity US it is necessary to expose cells to a sonosensitizer, i.e. a substance that displays a property of sensitizing cells to US. Many photosensitizers, for example hematoporphyrin and its derivatives, act also as sonosensitizers. Low-intensity sonication of cells causes cavitation and other direct acoustic effects that, in the presence of a sonosensitizer, evoke cytotoxic oxygen free radicals. An ideal sonosensitizer should be preferentially taken up and retained 
by cancer cells, and display no significant toxicity toward normal tissue. Unlike PDT, which due to limited penetration of light through tissues is applicable only to superficially located tumors, SDT might be used to treat deeply seated cancers $[12,16]$.

One of the photosensitizers used in PDT and potentially useful also for SDT is 5-aminolevulinic acid (ALA), a natural precursor of protoporphyrin IX (PpIX). Due to peculiar metabolic abnormality usually associated with cancer, PpIX is preferentially accumulated in cancer cells, in particular in cells of malignant gliomas, therefore ALA is capable of sensitizing them selectively [4,13]. Development of SDT is particularly awaited for the most malignant glioma, glioblastoma multiforme, which infiltrate brain, cannot be totally removed by surgery and escape radio- and chemotherapy, therefore it recurs and in the majority of cases is lethal within less than 2 years [3].

In the previous study [8] we developed an experimental arrangement for investigating effects of sonication on glioma cells in vitro and used this system to determine a relationship between US energy delivered to the rat C 6 glioma cells in vitro and their vitality; we also established the threshold exposure time that does not induce thermal effects which would be directly cytotoxic. The next step in the translational development of SDT for gliomas should be experiments with glioma cells implanted orthotopically into brains of experimental animals. C6 rat glioma cells implanted to the rat brain have been extensively used as a rat model of human malignant gliomas, but these cells evoke immune response which restricts their infiltrative growth [1]. Therefore for further studies we chose the RG2 rat glioma cell line which is not immunogenic when implanted to rats of Fisher or Wistar strain and displays a highly infiltrative pattern of growth, reminiscent of human glioblastoma multiforme [15].

\section{Material and methods \\ Cell culture and reagents}

The rat RG2 glioma cells were obtained from the cell bank of the American Type Culture Collection (Manassas, VA). The cells were cultured in Petri's dishes in a Dulbecco's modified Eagle's medium (DMEM, Gibco, Life Technologies Inc., USA) supplemented with a $10 \%$ fetal bovine serum (FBS, Hyclone, USA) and $1 \%$ antibiotics (penicillin and streptomycin). The cells were maintained at $37^{\circ} \mathrm{C}$ in a humidified atmosphere with $5 \% \mathrm{CO}_{2} / 95 \%$ air in incubator (Esco). In each experiment cells were used after $24 \mathrm{~h}$ of growth.

5-Aminolevulinic acid, 3-(4,5-dimethylthiazol-2-yl)2,5-diphenyl tetrazolium bromide and other chemicals used were purchased from Sigma-Aldrich (Germany), unless specified otherwise.

\section{Experimental set-up for sonodynamic therapy}

To examine the impact of ALA-mediated SDT therapy on the tested cells in vitro the experimental setup shown in Figure 1 was used.
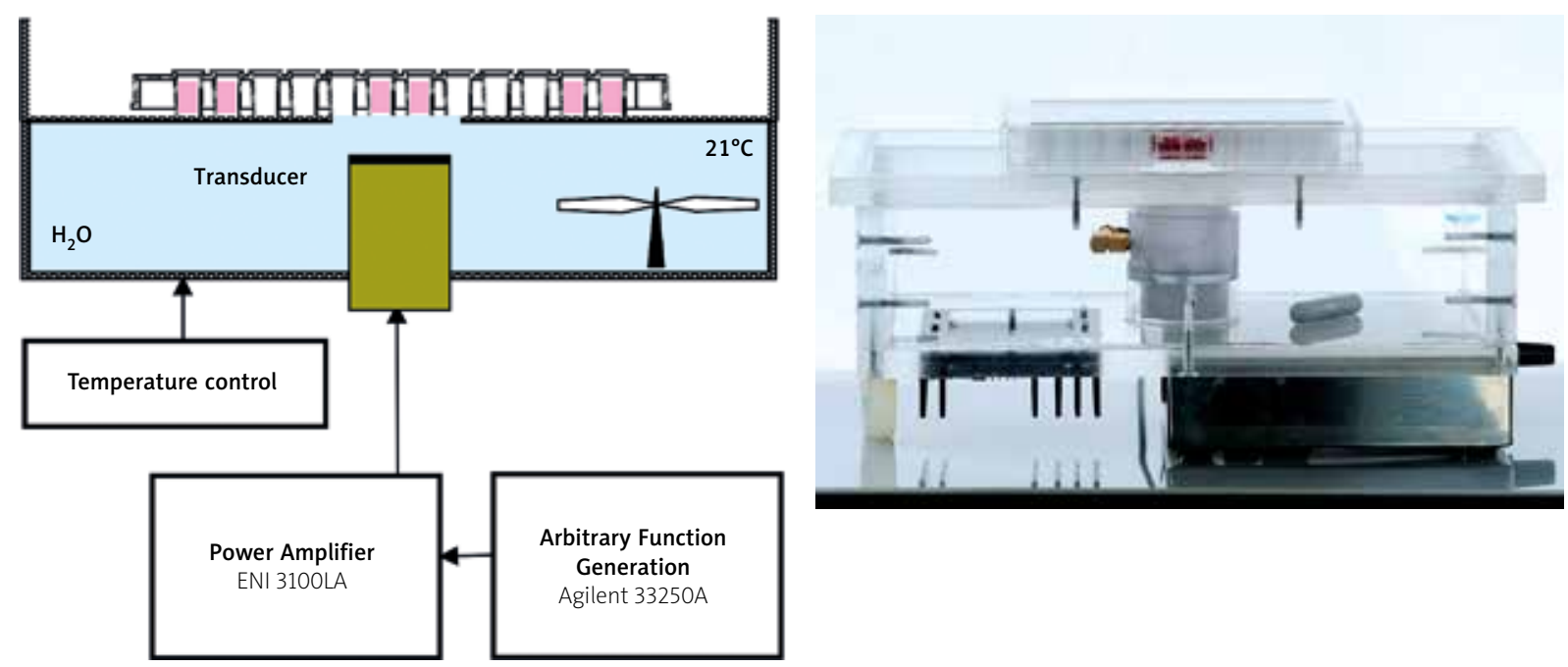

Fig. 1. The experimental arrangement (left) for sonification of rat RG2 glioma cells and its photo (right). 
To eliminate the temperature rise in the region of cells caused by the overheating of the US source, the transducer was mounted in the water bath coaxially with wells. The cells cultured on the bottom of wells were sonicated from the bottom of 96-well plates submerged in the water bath, with temperature set to $21^{\circ} \mathrm{C}$.

Ultrasonic waves were generated with a planar circular transducer made of a power piezoceramics Pz28 (Meggitt, Kvistgaard, Denmark) with the resonance frequency of $1 \mathrm{MHz}$ and diameter of $25 \mathrm{~mm}$. The transducer had neither a back load nor a quarterwavelength matching layer and was excited by 1000 -cycle sinusoidal pulses with a resonance frequency, 0.4 duty-cycle and varied voltage. The electrical pulses were generated by an arbitrary function generator Agilent 33250 (Colorado Springs, USA) and amplified with a power amplifier ENI 3100L (ENI, Rochester, NY, USA). The average acoustic power of the generated beam was varied from $2 \mathrm{~W}$ to $6 \mathrm{~W}$ at $2 \mathrm{~W}$ (initial intensity $I_{\text {SATA }}$ was varied between 0.5 and $1.5 \mathrm{~W} / \mathrm{cm}^{2}$ ) and measured using Ultrasound Power Meter UPMDT-10AV (Ohmic Instruments Co., Easton, USA). These specific ultrasound field parameters were selected to induce no thermal lethality of the cells tested.

\section{Determination of acoustic parameters of pulsed ultrasonic beams}

The acoustic properties of the generated pulsed pressure (intensity) beams were determined on the

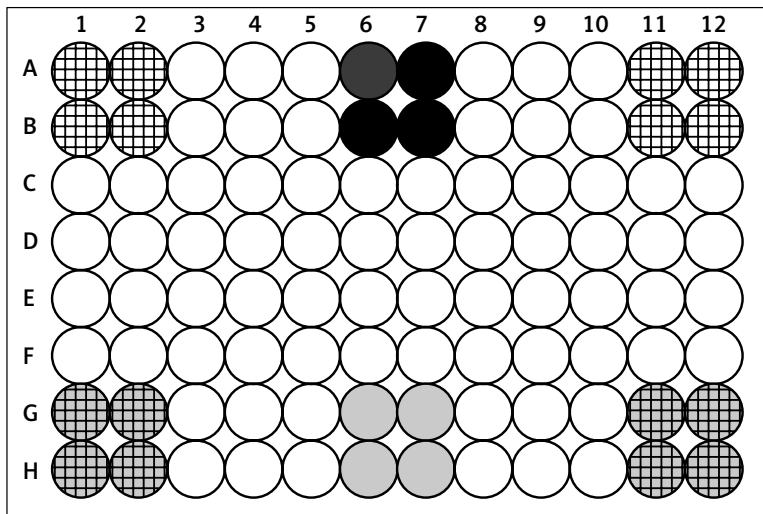

Fig. 2. Schematic arrangement of 4 groups of wells in each plate according to the kind of factor impacting cells: white - empty wells, black - control wells, gray - 5-aminolevulinic acid (ALA) alone wells, grid on a white background ultrasound (US) alone wells, grid on a gray background - ALA + US wells. basis of preliminary measurements in water. First, the transducer excitation voltage, providing generation of the beam with the selected average acoustic power measured by the Ultrasound Power Meter, was determined. As mentioned above, the transducer was excited by 1000-cycle sinusoidal pulses generated from an arbitrary function generator Agilent 33250 and amplified by the power amplifier ENI3100LA. The source pressure amplitude and the initial intensity $I_{\text {SATA }}$ of the generated beam for each voltage applied was determined by two methods: (1) using the measurements of the averaged radial pressure distribution near the transducer radiating surface using the calibrated $0.2 \mathrm{~mm}$ needle hydrophone S/N1661 (Precision Acoustics, Dorchester, UK) and (2) using the measurements of the average acoustic power using Ultrasound Power Meter UPM-DT-10AV (Ohmic Instruments Co., Easton, USA). The measurements by the needle hydrophone were carried out laterally at the axial distance of $1 \mathrm{~mm}$ from the transducer surface for the tone bursts with duration of $8 \mu \mathrm{s}$ and PRF of $0.1 \mathrm{kHz}$. The RMS value for the pulse duration was recorded with a LeCroy 62xi oscilloscope. The sensitivity of the needle hydrophone for the frequency used was equal to $59.7 \mathrm{mV} / \mathrm{MPa}$. The convergence of the source pressure amplitudes obtained by the two methods was within $4.4 \%$ and of the initial intensities or powers was within $9 \%$.

The spatial acoustic pressure distributions in the ultrasonic beams used were measured in water under free field conditions using broadband bilaminar membrane PVDF hydrophone (with active electrode of $0.5 \mathrm{~mm}$ in diameter).

\section{Sonodynamic therapy protocols}

The rat RG2 glioma cells were seeded on the bottom of 24 wells ( $6 \times 4$ wells) in 96-well polystyrene plates (Cellstar 96 Well Cell Culture Plate, Greiner Bio-One, USA), as shown in Figure 2.

$200 \mu \mathrm{l}$ of the cell suspension in DMEM medium supplemented with a $10 \%$ FBS, containing the same number of cells $\left(2 \times 10^{4}\right)$ were introduced to each well. After $24 \mathrm{~h}$ incubation at $37^{\circ} \mathrm{C}$ the medium was changed to DMEM without FBS or to DMEM without FBS but with ALA, and the cells were exposed for 3 min to ultrasound. During sonication a bottom of each plate was immersed in a water bath with temperature set to $21^{\circ} \mathrm{C}$. The thickness of the bottom of polystyrene plates was about $1 \mathrm{~mm}$. 
The wells in each plate were allocated to 4 groups: 1) No exposure (Control); 2) Exposure to ALA; 3) Exposure to US and 4) Exposure to ALA + US. For ALA and ALA + US experiments the cells were incubated for $6 \mathrm{~h}$ in serum-free DMEM with $100 \mu \mathrm{M}$ ALA to give them time to take up ALA and convert it to protoporphyrin IX. For the Control and US only experiments the same amount of DMEM was used. In the US and ALA + US experiments the cells were sonicated by pulsed ultrasound at a resonance frequency of $1 \mathrm{MHz}$ and acoustic power varied from $2 \mathrm{~W}$ to $6 \mathrm{~W}$ (spatial-averaged temporal-averaged intensity $\left[I_{\text {SATA }}\right]$ in the region of cells varied from 0.5 to $1.5 \mathrm{~W} / \mathrm{cm}^{2}$ ) during $3 \mathrm{~min}$ exposure. As demonstrated in the previous report [8] for such intensity levels and exposure time lethal thermal effects in the region of cells are not reached. After the treatment procedure the cells were re-suspended in fresh DMEM and subjected to further analyses.

\section{Cell viability detection}

To evaluate the effects of ALA and/or US on the rat RG2 glioma cells viability, a colorimetric 3-(4,5-dimethylthiazol-2-yl)-2,5-diphenyl tetrazolium bromide (MTT) assay was used. Following exposure to $\mathrm{ALA}$ and/or US the cells were incubated at $37^{\circ} \mathrm{C}$ for $24 \mathrm{~h}$, then $15 \mu \mathrm{l}$ solution of MTT at a concentration of $5 \mathrm{mg} / \mathrm{ml}$ was added to $150 \mu \mathrm{l}$ of culture medium in each of these wells (final concentration of MTT was $0.5 \mathrm{mg} / \mathrm{ml}$ ). After $3 \mathrm{~h}$ incubation the media were removed. The formazan crystals were dissolved in $200 \mu \mathrm{l}$ of dimethyl sulfoxide and this solution was added to each well. The absorbance of cells at $570 \mathrm{~nm}$ was measured using an Epoch micro-plate reader (Bio-Tek, ELX800, USA) in relation to the reference value at $630 \mathrm{~nm}$. The viability of treated cells was determined by comparing to the untreated ones in the Control group.

\section{Cell morphology and visualization of apoptosis}

After the experiments and $24 \mathrm{~h}$ incubation the cells were stained by the Hoechst 33342 Nuclear Staining Dye (Invitrogen) with a concentration of $1 \mathrm{\mu g} / \mathrm{ml}$ at $37^{\circ} \mathrm{C}$ for $10 \mathrm{~min}$. Nuclear Morphology of cell nuclei (chromatin condensation, presence of apoptotic bodies) was evaluated under an inverted fluorescence microscope IX81 Cell R equipped with a LUCPlanFLN objective (Olympus).

\section{Statistical analysis}

Statistical evaluation of data was performed using Graph-Pad Prism version 6.04 for Windows (GraphPad Software, San Diego, CA, USA). One way ANOVA was followed by Bonferroni's multiple comparisons post hoc test. Differences were considered significant when $p<0.05$ vs Control $(n=8)$. There were performed 2 independent MTT experiments with 8 repeats as well as 2 independent experiments of staining by Hoechst with 4 repeats.

\section{Results}

\section{Enhancement of ultrasound induced cell killing during ALA-mediated SDT}

As shown in Figure 3 at the exposure to US of $2 \mathrm{~W}$ cell survival for ALA + US group did not significantly differ from the respective controls (ALA alone), indicating no sonosensitization. However, when $6 \mathrm{~W}$ ultrasound was used, the cytotoxic effect in ALA + US group after $24 \mathrm{~h}$ was significant (see Fig. 4).

\section{Changes in morphology of cell nuclei}

The changes in cellular morphology, quantity of cells and chromatin condensation/apoptosis induced by ALA-mediated SDT on rat RG2 glioma cells depending on the acoustic power of ultrasound used are shown in Figures 5 and 6.

\section{Discussion}

In the present study, rat RG2 glioma cells were exposed to 5-aminolevulinic acid and afterwards son-

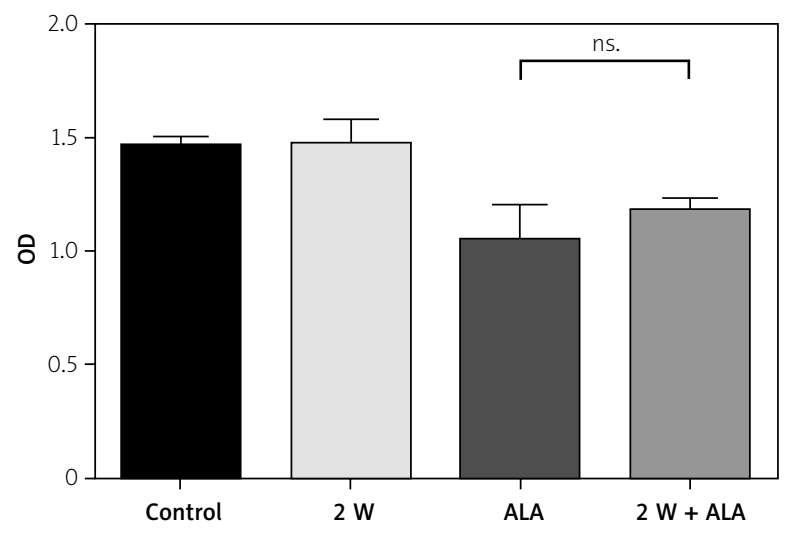

Fig. 3. Cytotoxic effect of 5-aminolevulinic acid and ultrasound (ALA + US) (2 W, 3 min. exposure) on viability of rat RG2 glioma cells after $24 \mathrm{~h}$ (mean \pm SD, $n=8$ ). 


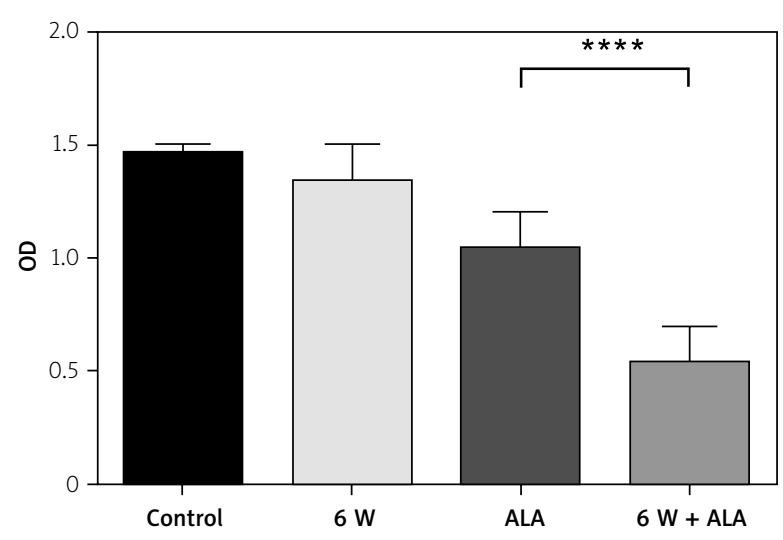

Fig. 4. Cytotoxic effect of ultrasound 5-aminolevulinic acid and ultrasound (ALA + US) (6 W, 3 min. exposure) on viability of rat RG2 glioma cells after $24 \mathrm{~h}$ (mean $\pm \mathrm{SD}, n=8$ ).

icated with pulsed low intensity ultrasound waves. Our aim was to look for the sonodynamic effect, and in particular for signs of SDT-mediated apoptotic cell death. As mentioned in the introduction, the RG2 cells seem to be better suited for preclinical research using orthotopic implantation than the $\mathrm{C} 6$ cells because they are less immunogenic and their growth in brain is more infiltrative. Nevertheless, the RG2 glioma cells are much less used in research than the C6 glioma cells. In particular, whereas several papers described reactions of the $C 6$ cells in vivo to
ALA-mediated PDT $[2,7,17]$ and SDT $[8,9,14]$, we were unable to find any report on experiments with the RG2 cells and either SDT or PDT.

Currently the mechanism of sonosensitization of glioma cells by ALA is poorly understood. Results obtained in the present study provide evidence for ALA-mediated sonosensitization of RG2 rat glioma cells in vitro, but this effect was evident only when the cells pre-incubated with ALA were sonicated by US with intensity of $6 \mathrm{~W}$, which we consider the upper limit of US dose that is not directly lethal to the cells due to rise in temperature. In the present experiments, neither the concentration of PpIX, nor the amount of free radicals in the cells were measured, therefore no direct evidence can be presented for a mechanism of sonosensitization. While other mechanisms of cytotoxicity such as necrosis or autophagy cannot be excluded, the present experiments provided evidence of apoptosis being the major event evoked by sonosensitization of RG2 cells with ALA.

The SDT technique is tested not only in vitro, but also in vivo. A selective effect of low intensity US against $\mathrm{C} 6$ glioma cells implanted to the rat brain was achieved for the first time with the use of Rose Bengal dye given intravenously as a sonosensitizer [10]. Since then two other publications have reported on experiments in which ALA-mediated STD were tested in vivo on orthotopically transplanted C6 glioma $[5,11]$. In
Control
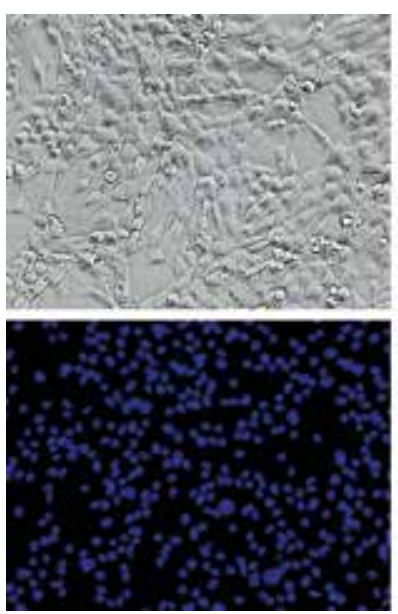

ALA
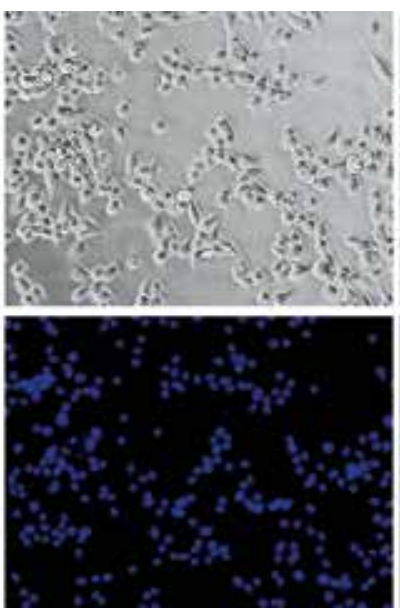

US ( 2 W)
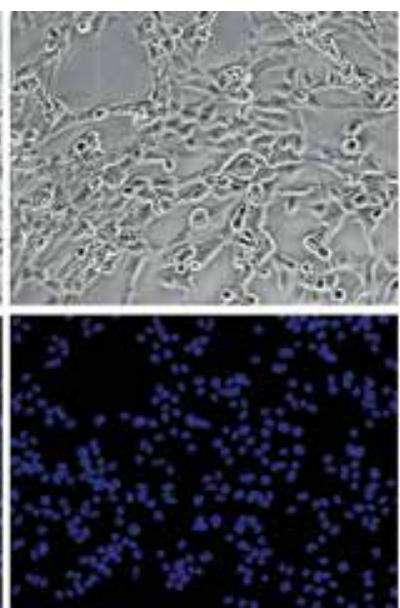

$A L A+U S(2 W)$
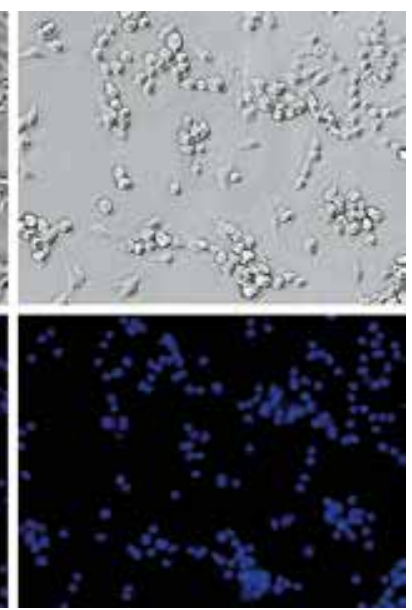

Fig. 5. The morphology, quantity and chromatin condensation/apoptosis of RG2 rat glioma cells $24 \mathrm{~h}$ after 5-aminolevulinic acid (ALA)-mediated SDT (3-minute exposure to $2 \mathrm{~W}$ ultrasound). The top images show cell cultures visualized by a light microscope (contrast phase). The bottom images show the nuclear morphology (Hoechst 33352 staining, fluorescence microscopy). There are slight differences in the cell number and their morphological features between ALA, ultrasound (US) (2 W) and ALA + US (2 W) groups. 

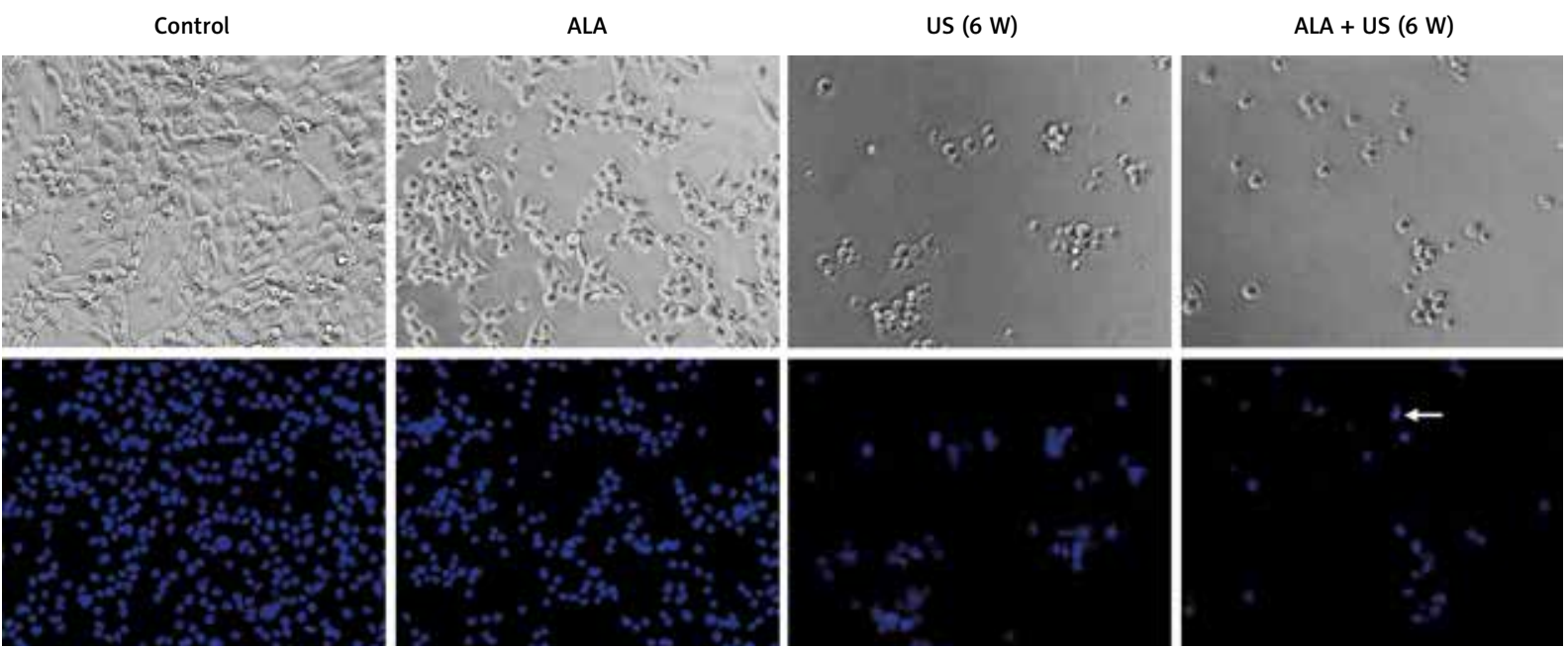

Fig. 6. The morphology, quantity and chromatin condensation/apoptosis of RG2 rat glioma cells $24 \mathrm{~h}$ after 5-aminolevulinic acid (ALA)-mediated SDT (3-minute exposure to $6 \mathrm{~W}$ ultrasound). The top images show cell cultures visualized by a light microscope (contrast phase). The bottom images show the nuclear morphology (Hoechst 33352 staining, fluorescence microscopy). There are noticeable differences in the cell number and their morphological changes between ALA alone, ultrasound (US) (6 W) alone and ALA + US (6 W) groups. Arrows point to nuclei with chromatin condensation, arrowheads show apoptotic bodies.

these papers SDT-induced decreases in gross tumor volume evaluated post-mortem were shown.

Effective therapy of human malignant gliomas would require elimination of clonogenic glioma cells which spread beyond tumor margins that are visible during surgery or visualized by radiological techniques [6]. These cells, some of which are located at a considerable distance from gross tumor margins, cause glioma recurrence. While it has already been shown that ALA-mediated SDT applied to intracranially growing C6 glioma in rat can cause gross tumor margins to shrink, it remains to be investigated whether this technique can also eliminate clonogenic glioma cells located at a distance from the tumor. For such study RG2 glioma cell line would certainly be a better choice.

\section{Disclosure}

Authors report no conflict of interest.

\section{References}

1. Barth RF, Kaur B. Rat brain tumor models in experimental neurooncology: the C6, 9L, T9, RG2, F98, BT4C, RT-2 and CNS-1 gliomas. J Neurooncol 2009; 94: 299-312.

2. Beck TJ, Burkanas M, Bagdonas S, Krivickiene Z, Beyer W, Sroka R, Baumgartner, Rotomskis R. Two-photon photodynamic therapy of $C 6$ cells by means of 5-aminolevulinic acid induced protoporphyrin IX. J Photochem Photobiol B 2007; 87: 174-182.
3. Cuddapah VA, Robel S, Watkins S, Sontheimer H. A neurocentric perspective on glioma invasion. Nat Rev Neurosci 2014; 15 : 455-465.

4. Grieb P. 5-Aminolevulinic acid (ALA) and its applications in neurosurgery. Neurol Neurochir Pol 2004; 38: 201-207.

5. Jeong EJ, Seo SJ, Ahn YJ, Choi KH, Kim KH, Kim JK. Sonodynamically induced antitumor effects of 5-aminolevulinic acid and fractionated ultrasound irradiation in an orthotopic rat glioma model. Ultrasound Med Biol 2012; 38: 2143-2150.

6. Ji M, Lewis S, Camelo-Piragua S, Ramkissoon SH, Snuderl M, Venneti S, Fisher-Hubbard A, Garrard M, Fu D, Wang AC, Heth JA, Maher CO, Sanai N, Johnson TD, Freudiger CW, Sagher O, Xie XS, Orringer DA. Detection of human brain tumor infiltration with quantitative stimulated Raman scattering microscopy. Sci Transl Med 2015; 7: 309ra163.

7. Kamoshima Y, Terasaka S, Kuroda S, Iwasaki Y. Morphological and histological changes of glioma cells immediately after 5-aminolevulinic acid mediated photodynamic therapy. Neurol Res 2011; 33: 739-746.

8. Kujawska T, Secomski W, Bilmin K, Nowicki A, Grieb P. Impact of thermal effects induced by ultrasound on viability of rat C6 glioma cells. Ultrasonics 2014; 54: 1366-1372.

9. Li JH, Chen ZQ, Huang Z, Zhan Q, Ren FB, Liu JY, Yue W, Wang Z. In vitro study of low intensity ultrasound combined with different doses of PDT: Effects on C6 glioma cells. Oncol Lett 2013; 5: 702-706.

10. Nonaka M, Yamamoto M, Yoshino S, Umemura S, Sasaki K, Fukushima T. Sonodynamic therapy consisting of focused ultrasound and a photosensitizer causes a selective antitumor effect in a rat intracranial glioma model. Anticancer Res 2009; 29: 943-950.

11. Ohmura T, Fukushima T, Shibaguchi H, Yoshizawa S, Inoue T, Kuroki M, Sasaki K, Umemura S. Sonodynamic therapy with 
5-aminolevulinic acid and focused ultrasound for deep-seated intracranial glioma in rat. Anticancer Res 2011; 31: 2527-2533.

12. Shibaguchi H, Tsuru H, Kuroki M, Kuroki M. Sonodynamic cancer therapy: a non-invasive and repeatable approach using low-intensity ultrasound with a sonosensitizer. Anticancer Res 2011; 31: 2425-2429.

13. Tetard MC, Vermandel M, Mordon S, Lejeune JP, Reyns N. Experimental use of photodynamic therapy in high grade gliomas: a review focused on 5-aminolevulinic acid. Photodiagnosis Photodyn Ther 2014; 11: 319-330.

14. Tserkovsky DA, Alexandrova EN, Chalau VN, Istomin YP. Effects of combined sonodynamic and photodynamic therapies with photolon on a glioma C6 tumor model. Exp Oncol 2012; 34 332-335.

15. Weizsäcker M, Nagamune A, Winkelströter R, Vieten H, Wechsler W. Radiation and drug response of the rat glioma RG2. Eur J Cancer Clin Oncol 1982; 18: 891-895.

16. Wood AK, Sehgal CM. A review of low-intensity ultrasound for cancer therapy. Ultrasound Med Biol 2015; 41: 905-928.

17. Wu SM, Ren QG, Zhou MO, Peng Q, Chen JY. Protoporphyrin IX production and its photodynamic effects on glioma cells, neuroblastoma cells and normal cerebellar granule cells in vitro with 5-aminolevulinic acid and its hexylester. Cancer Lett 2003; 200: 123-131 